\title{
Correction to: A Proposal of Neural Networks with Intermediate Outputs
}

Billy Peralta (D), Juan Reyes, Luis Caro (D), and Christian Pieringer (D)

\section{Correction to:}

Chapter "A Proposal of Neural Networks with Intermediate Outputs" in: A. Morales et al. (Eds.): Pattern Recognition and Image Analysis, LNCS 11867, https://doi.org/10.1007/978-3-030-31332-6_18

The original version of this chapter was revised and the spelling of Billy Peralta's affiliation has been changed to "Universidad Andres Bello". 